\title{
Screening of Mungbean Genotypes against Mungbean Yellow Mosaic Virus Disease
}

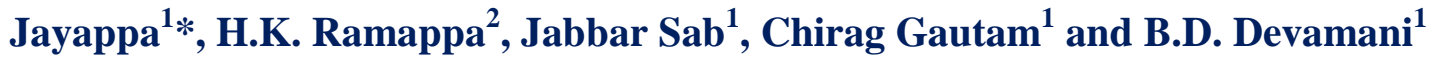 \\ ${ }^{1}$ Department of Plant Pathology, College of Agriculture, UAS, GKVK, \\ Bengaluru-560065, Karnataka, India \\ ${ }^{2}$ AICRP on Pigeonpea ZARS, UAS, GKVK Bengaluru-560065 Karnataka, India \\ *Corresponding author
}

\begin{tabular}{|c|c|}
\hline & A B S T R A C T \\
\hline $\begin{array}{l}\text { Mungbean, } \\
\text { MYMV, } \\
\text { Screening, } \\
\text { Genotype, } \\
\text { Whitefly. }\end{array}$ & \multirow{3}{*}{$\begin{array}{l}\text { Hundred genotypes of mungbean were screened against Mungbean Yellow } \\
\text { Mosaic Virus (MYMV) disease. Among hundred genotypes screened, three } \\
\text { genotypes namely LGG-593, KMP-24, and KMP-40 were found moderately } \\
\text { resistant, nineteen genotypes viz., KMP-4, KMP-22, VBN-2-3, LGG-594, } \\
\text { MGG-40-1, JNG-13, TK-6-1, BGG-1, VBN-2-3, LGG-410, LGG-410, KKM- } \\
\text { 3-1, ICC-12947, LGG-592, KMP-1, KMP-22, KMP-23, KMP-17 and KMP-4 } \\
\text { found moderately susceptible, sixty-six genotypes found susceptible and } \\
\text { twelve genotypes were found highly susceptible. Maximum whiteflies } \\
\text { population per trifoliate leaf was recorded in highly susceptible genotypes and } \\
\text { positive correlation between vector population and disease incidence was also } \\
\text { observed. }\end{array}$} \\
\hline Article Info & \\
\hline $\begin{array}{l}\text { Accepte } \\
17 \text { April } \\
\text { Availab } \\
\text { 12 May }\end{array}$ & \\
\hline
\end{tabular}

\section{Introduction}

Mungbean (Vigna radiata $\mathrm{L}$.) is also known as mung, moong, mungo. In India, the name greengram is more commonly used than mungbean (Chatterjee and Randhawa, 1952). It is third most important pulse crop of India after chickpea and pigeonpea.

Mungbean can grow in all three seasons in India, viz., Kharif (July- Oct), Rabi (SeptDec) and summer (March- June). It is grown under rainfed condition during Kharif and on residual moisture during Rabi in eastern and southern part of the country. However, Kharif season crop occupies maximum area of its cultivation.
Intercropping with sorghum, pearl-millet, maize, cotton, castor, pigeonpea etc., are popular. India alone contributes $54 \%$ of world production and covers $65 \%$ of the world acreage (Singh, 2011). It is grown in intercropping, catch cropping and relay cropping systems because of its short duration (less than 60 days).

In India, mungbean is grown in an area of 3.02 lakh ha with production of 1.50 lakh tons and productivity of $498 \mathrm{~kg} / \mathrm{ha}$. Important mungbean growing states are Rajasthan, Maharashtra, Karnataka, Andhra Pradesh, Odisha, Tamil Nadu and Uttar Pradesh 
(Anon, 2012). The standard worldwide yield of mungbean is very low $(389 \mathrm{~kg} / \mathrm{ha})$ and its production has not increased over the year. The main reason for low yield is the susceptibility of the crop to insects, weeds and diseases caused by fungus, virus and bacteria. Among these, the viruses are the most important group of plant pathogens affecting the production of the crop. They cause severe diseases and economic losses in mungbean and reducing seed yield and quality (Kang et al., 2005).

As many as 12 viral diseases are reported on mungbean crop viz., Mungbean yellow mosaic virus (MYMV) disease, Bean yellow mosaic virus (BYMV) disease, Southern bean mosaic virus (SBMV) disease, Leaf crinkle virus(LCV) disease, Mungbean mosaic virus (MMV) disease, Cowpea aphid-borne virus (CABV) disease, Cucumber yellow mosaic virus (CMV) disease, Alfalfa mosaic virus (AMV) disease, Leaf curl virus (LCV) disease, Bean common mosaic virus (BCMV) disease, Cowpea yellow mosaic virus (CYMV) disease, Blackeye cowpea mosaic virus (BLCMV) disease.

Among these, occurrence of MYMV is the major constraint in successful cultivation of mungbean. Mungbean yellow mosaic virus is a geminivirus belongs to Family Geminiviridae, Subgroup - II, Genus: Begomovirus containing ssDNA and spread by Bemicia tabaci, considered as the most important and destructive viral pathogen in many parts of India (Saikia and Muniyappa, 1989).

Plant resistance for MYMV is rare and has not been actively pursued in breeding programs, hence, in this present study, germplasm lines have been screened for resistance against MYMV, that may be useful to reduce the impact of the disease in Mungbean crop.

\section{Materials and Methods}

A field experiment was conducted in the experimental plots, maintained at ZARS, GKVK, Bengaluru, India. Hundred mungbean genotypes including varieties were used for screening.

Plant spacing of $30 \times 10 \mathrm{~cm}$ with a row length of $2.5 \mathrm{~m}$ in RCBD design with two replications and all the package of practices were followed for raising the crop. Plants were exposed to natural infection. Per cent disease incidence of MYMV disease in each genotype/ variety was calculated based on disease scale and ratings.

Per cent Disease Incidence $(\mathrm{PDI})=$

Number of infected plants

Total number of plants observed X 100

The genotypes were later grouped into different categories based on 0 to 9 scale (Mayee and Datar, 1986) as given in table 1.

\section{Results and Discussion}

The objective was to identify source of resistance against the MYMV disease. The grouping of genotypes based on per cent incidence of the disease in each genotype/variety at pre-flowering stage was done as described in 'Materials and Methods'. The results are presented in table 2 .

Mungbean genotypes including varieties were screened against MYMV under field condition with natural infection by Bemicia tabaci. Disease rating scale was followed as given by Mayee and Datar (1986).

Mungbean yellow mosaic virus disease incidence varied from 8.03 per cent in KMP24 to 78.12 per cent in KMP-47 with disease 
reaction of moderately resistant and highly susceptible respectively (Table 3).

Only three genotypes such as KMP-24, KMP40 and LGG-593 with 8.03 per cent, 8.26 per cent and 11.06 per cent respectively were found moderately resistant as shown in Plate1 ( $\mathrm{a}, \mathrm{b}$ and $\mathrm{c})$. Yellow mottling and discolouration of leaves were observed in all these lines.

Sixteen genotypes found to be moderately susceptible such as KMP-4, KMP-22, VBN2-3, LGG-594, MGG-40-1, JNG-13, TK-6-1, BGG-1， VBN-2-3， LGG-410， LGG-410, KKM-3-1，ICC-12947，LGG-594，KMP-1, KMP-23, KMP-17 and KMP-4 having per cent disease incidence of 15.58, 16.66, 13.04, $15.64,16.91,14.06,17.85,15.48,13.04$, $13.63,21.12,14.33,08.33,18.25,13.18$, 20.18 and 15.58 respectively and infected plants shown symptoms of leaf puckering. Mohan et al., (2014) was also reported similar results wherein, he screened 120 germplasm lines under field condition at two locations during Kharif 2013 by planting infector rows along with the test entries. Results revealed that most of the genotypes were categorized as moderately susceptible to highly susceptible in both the locations. None of the test entries appeared to be immune.

Sixty-nine genotypes were showed susceptible reaction with the range of 21.07 to 50.23 per cent. TM-97-55, GREEN DIAMOND，LGG-572，KKM-3，KKM-3-5, PUSA-9537, MGG-347, LGG-1, KMP-6, ML-4, PDM-84-578, JNG-21, PUSA BAISAKI-1, GNJ-5, KKM-3-10, VGG-04149, NM-94, JNG-5, JNG-18, NM-97, JNG14-1, LGG-595, JNG-2, PM-115, LGG-920, 2KM-139, TURUVEKERE LOCAL, VGG04-011, LGG-596, JNG-12, JNG-19, 2KM165, COGG-954, 2KM-165, 2KM-137, TM962, 2KM-102, VGG-07-3, 2KM-101, VBN(Gg)-2, PM-110, VBN(g)-3, KMP-41, KMP-46, KMP-30, KMP-42, KMP-12, KMP27, KMP-19, KMP-9, KMP-2, KMP-45, KMP-7, KMP-28, KMP-21, KMP-32, KMP35, KMP-48, KMP-18,KMP-25, KMP11,KMP-26, KMP-44, KMP-14, KMP-52, KMP-5, KMP-39, KMP-6 and KMP-34 lines were found susceptible to MYMV. Pronounced yellow mottling and discolouration of leaves, reduction in leaf size and stunting of plants were observed, these results were also correlated with Singh et al., (1996).

Table.1 Disease scale referred for grouping of genotypes against MYMV disease (Mayee and Datar, 1986)

\begin{tabular}{|c|l|l|}
\hline Scale & \multicolumn{1}{|c|}{ Description } & \multicolumn{1}{c|}{ Category } \\
\hline 0 & No plants showing any symptom & Immune (I) \\
\hline 1 & $1 \%$ or less plants exhibiting symptoms & Resistant (R) \\
\hline 3 & $1-10 \%$ plants exhibiting symptoms & Moderately resistant ( MR) \\
\hline 5 & $11-20 \%$ plants exhibiting symptoms & Moderately susceptible (MS) \\
\hline 7 & $21-50 \%$ plants exhibiting symptoms & Susceptible (S) \\
\hline 9 & $51 \%$ plants exhibiting symptoms & Highly susceptible (HS) \\
\hline
\end{tabular}


Table.2 Reaction of Mungbean genotypes against MYMV

\begin{tabular}{|c|c|c|c|c|c|c|c|c|c|}
\hline Sl. No & Genotypes & $\begin{array}{c}\text { Per cent } \\
\text { disease } \\
\text { incidence }\end{array}$ & $\begin{array}{c}\text { Disease } \\
\text { Reaction* }\end{array}$ & Scale & SI. No & Genotypes & $\begin{array}{c}\text { Per cent } \\
\text { disease } \\
\text { incidence }\end{array}$ & $\begin{array}{c}\text { Disease } \\
\text { Reaction* }\end{array}$ & Scale \\
\hline 1 & KMP-34 & 31.75 & $\mathrm{~S}$ & 7 & 26 & KMP-23 & 13.18 & MS & 5 \\
\hline 2 & KMP-13 & 67.09 & HS & 9 & 27 & KMP-2 & 32.06 & $\mathrm{~S}$ & 7 \\
\hline 3 & KMP-6 & 46.51 & $\mathrm{~S}$ & 7 & 28 & KMP-36 & 53.75 & HS & 9 \\
\hline 4 & KMP-33 & 55.84 & HS & 9 & 29 & KMP-42 & 41.81 & $\mathrm{~S}$ & 7 \\
\hline 5 & KMP-39 & 42.14 & $S$ & 7 & 30 & KMP-12 & 40.92 & $\mathrm{~S}$ & 7 \\
\hline 6 & KMP-5 & 26.63 & $S$ & 7 & 31 & KMP-27 & 48.90 & $\mathrm{~S}$ & 7 \\
\hline 7 & KMP-52 & 49.54 & $\mathrm{~S}$ & 7 & 32 & KMP-19 & 24.9 & $\mathrm{~S}$ & 7 \\
\hline 8 & KMP-3 & 56.48 & HS & 9 & 33 & KMP-9 & 24.92 & $\mathrm{~S}$ & 7 \\
\hline 9 & KMP-14 & 41.10 & $\mathrm{~S}$ & 7 & 34 & KMP-22 & 16.66 & M S & 5 \\
\hline 10 & KMP-4 & 15.58 & MS & 5 & 35 & KMP-30 & 49.07 & $\mathrm{~S}$ & 7 \\
\hline 11 & KMP-44 & 43.04 & $\mathrm{~S}$ & 7 & 36 & KMP-40 & 8.26 & MR & 3 \\
\hline 12 & KMP-26 & 50.23 & $\mathrm{~S}$ & 7 & 37 & KMP-41 & 50.00 & $\mathrm{~S}$ & 7 \\
\hline 13 & KMP-47 & 78.12 & HS & 9 & 38 & KMP-46 & 47.18 & $\mathrm{~S}$ & 7 \\
\hline 14 & KMP-11 & 50.22 & $\mathrm{~S}$ & 7 & 39 & KMP-1 & 18.25 & MS & 5 \\
\hline 15 & KMP-25 & 46.63 & $\mathrm{~S}$ & 7 & 40 & KMP-24 & 8.03 & M R & 3 \\
\hline 16 & KMP-18 & 41.81 & $S$ & 7 & 41 & VBN(g)-3 & 44.52 & $S$ & 7 \\
\hline 17 & KMP-48 & 49.67 & $S$ & 7 & 42 & LGG-593 & 11.06 & MS & 5 \\
\hline 18 & KMP-17 & 20.18 & MS & 5 & 43 & VGG-07-3 & 37.50 & $\mathrm{~S}$ & 7 \\
\hline 19 & KMP-32 & 43.37 & $\mathrm{~S}$ & 7 & 44 & 2KM-101 & 46.82 & $\mathrm{~S}$ & 7 \\
\hline 20 & KMP-35 & 37.39 & $\mathrm{~S}$ & 7 & 45 & $\mathrm{VBN}(\mathrm{Gg})-2$ & 35.71 & $\mathrm{~S}$ & 7 \\
\hline 21 & KMP-21 & 28.5 & $\mathrm{~S}$ & 7 & 46 & PM-110 & 33.42 & $\mathrm{~S}$ & 7 \\
\hline 22 & KMP-20 & 58.69 & HS & 9 & 47 & 2KM-102 & 21.07 & $\mathrm{~S}$ & 7 \\
\hline 23 & KMP-45 & 39.28 & $\mathrm{~S}$ & 7 & 48 & ICC-12947 & 14.33 & MS & 5 \\
\hline 24 & KMP-7 & 31.88 & $\mathrm{~S}$ & 7 & 49 & 2KM-165 & 40.00 & $\mathrm{~S}$ & 7 \\
\hline 25 & KMP-28 & 35.71 & $\mathrm{~S}$ & 7 & 50 & 2KM-137 & 42.25 & $\mathrm{~S}$ & 7 \\
\hline
\end{tabular}


Int.J.Curr.Microbiol.App.Sci (2017) 6(5): 1746-1752

\begin{tabular}{|c|c|c|c|c|c|c|c|c|c|}
\hline Sl. No & Genotypes & $\begin{array}{c}\text { Per cent } \\
\text { disease } \\
\text { incidence }\end{array}$ & $\begin{array}{c}\text { Disease } \\
\text { Reaction* }\end{array}$ & Scale & Sl. No & Genotypes & $\begin{array}{c}\text { Per cent } \\
\text { disease } \\
\text { incidence }\end{array}$ & $\begin{array}{c}\text { Disease } \\
\text { Reaction* }\end{array}$ & Scale \\
\hline 51 & TM-962 & 30.73 & $\mathrm{~S}$ & 7 & 75 & KKM-3-10 & 21.12 & $\mathrm{~S}$ & 7 \\
\hline 52 & LGG-563 & 60.43 & HS & 9 & 76 & VGG-04-149 & 29.42 & $\mathrm{~S}$ & 7 \\
\hline 53 & JNG-19 & 40.52 & $\mathrm{~S}$ & 7 & 77 & NM-94 & 29.72 & $\mathrm{~S}$ & 7 \\
\hline 54 & $2 \mathrm{KM}-165$ & 38.00 & $\mathrm{~S}$ & 7 & 78 & JNG-5 & 38.36 & $\mathrm{~S}$ & 7 \\
\hline 55 & COGG-954 & 33.75 & $\mathrm{~S}$ & 7 & 80 & TK-6-1 & 17.85 & MS & 5 \\
\hline 56 & VGG-04-025 & 64.00 & HS & 9 & 81 & GNJ-5 & 35.11 & $\mathrm{~S}$ & 7 \\
\hline 57 & JNG-12 & 49.99 & $\mathrm{~S}$ & 7 & 82 & JNJ-13 & 14.06 & MS & 5 \\
\hline 58 & KKM-3-1 & 15.00 & MS & 5 & 83 & PUSA BAISAKI-1 & 46.73 & $\mathrm{~S}$ & 7 \\
\hline 59 & $\begin{array}{l}\text { TURUVEKERE } \\
\text { LOCAL }\end{array}$ & 44.92 & $\mathrm{~S}$ & 7 & 84 & MGG-40-1 & 16.91 & MS & 5 \\
\hline 60 & VGG-04-011 & 29.48 & $\mathrm{~S}$ & 7 & 85 & MGG-347 & 23.36 & $\mathrm{~S}$ & 7 \\
\hline 61 & LGG-596 & 48.00 & $S$ & 7 & 86 & JNG-16 & 58.46 & HS & 9 \\
\hline 62 & LGG-410 & 13.63 & MS & 5 & 87 & LGG-1 & 24.09 & $\mathrm{~S}$ & 7 \\
\hline 63 & VGG-112 & 51.28 & HS & 9 & 88 & KMP-6 & 43.33 & $\mathrm{~S}$ & 7 \\
\hline 64 & $2 \mathrm{KM}-139$ & 50.19 & $\mathrm{~S}$ & 7 & 91 & JNG-21 & 31.19 & $\mathrm{~S}$ & 7 \\
\hline 65 & VBN-2-3 & 13.04 & MS & 5 & 92 & VBN-2-3 & 12.96 & MS & 5 \\
\hline 66 & LGG-920 & 33.33 & $\mathrm{~S}$ & 7 & 93 & LGG-594 & 15.64 & MS & 5 \\
\hline 67 & BGG-1 & 15.48 & MS & 5 & 94 & LGG-593 & 08.33 & MR & 3 \\
\hline 68 & NG-14-1 & 50.00 & $\mathrm{~S}$ & 7 & 95 & KKM-3-5 & 24.93 & $\mathrm{~S}$ & 7 \\
\hline 69 & LGG-595 & 44.86 & $\mathrm{~S}$ & 7 & 96 & PUSA-9537 & 37.43 & $\mathrm{~S}$ & 7 \\
\hline 70 & JNG-2 & 27.77 & $\mathrm{~S}$ & 7 & 97 & $\mathrm{TM}-97-55$ & 27.30 & $\mathrm{~S}$ & 7 \\
\hline 71 & PM-115 & 31.15 & $S$ & 7 & 98 & KKM-3 & 43.51 & $\mathrm{~S}$ & 7 \\
\hline 72 & NM-97 & 43.34 & $\mathrm{~S}$ & 7 & 99 & LGG-572 & 33.46 & $\mathrm{~S}$ & 7 \\
\hline 73 & 2KM-164 & 79.78 & HS & 9 & 100 & GREEN DIAMOND & 47.39 & $\mathrm{~S}$ & 7 \\
\hline 74 & JNG-15 & 76.66 & $\mathrm{HS}$ & 9 & & & & & \\
\hline
\end{tabular}


Table.3 Grouping of Mungbean genotypes based on their reaction to MYMV

\begin{tabular}{|c|l|c|c|l|}
\hline S1. No & Reaction & Scale & $\begin{array}{c}\text { No. of } \\
\text { genotypes }\end{array}$ & Genotypes \\
\hline 1 & Immune & 0 & 0 & \multicolumn{1}{|c|}{-} \\
\hline 2 & Resistant & 1 & 0 & \\
\hline 3 & $\begin{array}{l}\text { Moderately } \\
\text { resistant }\end{array}$ & 3 & 3 & LGG-593, KMP-24 and KMP-40 \\
\hline 4 & $\begin{array}{l}\text { Moderately } \\
\text { susceptible }\end{array}$ & 5 & 16 & $\begin{array}{l}\text { KMP-04, KMP-22, LGG-594, MGG-40-1, JNG-13, TK-6-1, BGG-1, } \\
\text { LGG-410, VBN-2-3, KKM-3-1, ICC-12947, LGG-592, KMP-1, KMP- } \\
\text { 22, KMP-23 and KMP-17 }\end{array}$ \\
\hline 5 & Susceptible & 7 & 69 & $\begin{array}{l}\text { TM-97-55, GREEN DIAMOND, LGG-572, KKM-3, KKM-3-5, PUSA- } \\
\text { 9537, MGG-347, LGG-1, KMP-6, ML-4, PDM-84-578, JNG-21, PUSA } \\
\text { BAISAKI-1, GNJ-5, KKM-3-10, VGG-04-149, NM-94, JNG-5, JNG- } \\
18, \text { NM-97, JNG-14-1, LGG-595, JNG-2, PM-115, LGG-920, 2KM- } \\
\text { 139, TURUVEKERE LOCAL, VGG-04-011, LGG-596, JNG-12, JNG- } \\
\text { VGG-07-3, 2KM-101, VBN(Gg)-2, PM-110, VBN(g)-3, KMP-41, } \\
\text { KMP-46, KMP-30, KMP-42, KMP-12, KMP-27, KMP-19, KMP-9, } \\
\text { KMP-2, KMP-45, KMP-7, KMP-28, KMP-21, KMP-32, KMP-35, } \\
\text { KMP-48, KMP-18, KMP-25, KMP-11,KMP-26, KMP-44, KMP-14, } \\
\text { KMP-52, KMP-5, KMP-39, KMP-6 and KMP-34 }\end{array}$ \\
\hline 6 & Highly susceptible & 9 & 12 & $\begin{array}{l}\text { KMP-13, KMP-33, KMP-3, KMP-47, KMP-20, KMP-36, LGG-563, } \\
\text { VGG-04-025, VGG-112, 2KM-164, JNG-15 and JNG-16. }\end{array}$ \\
\hline
\end{tabular}

Plate.1 Reaction of Mungbean genotypes to MYMV in field condition

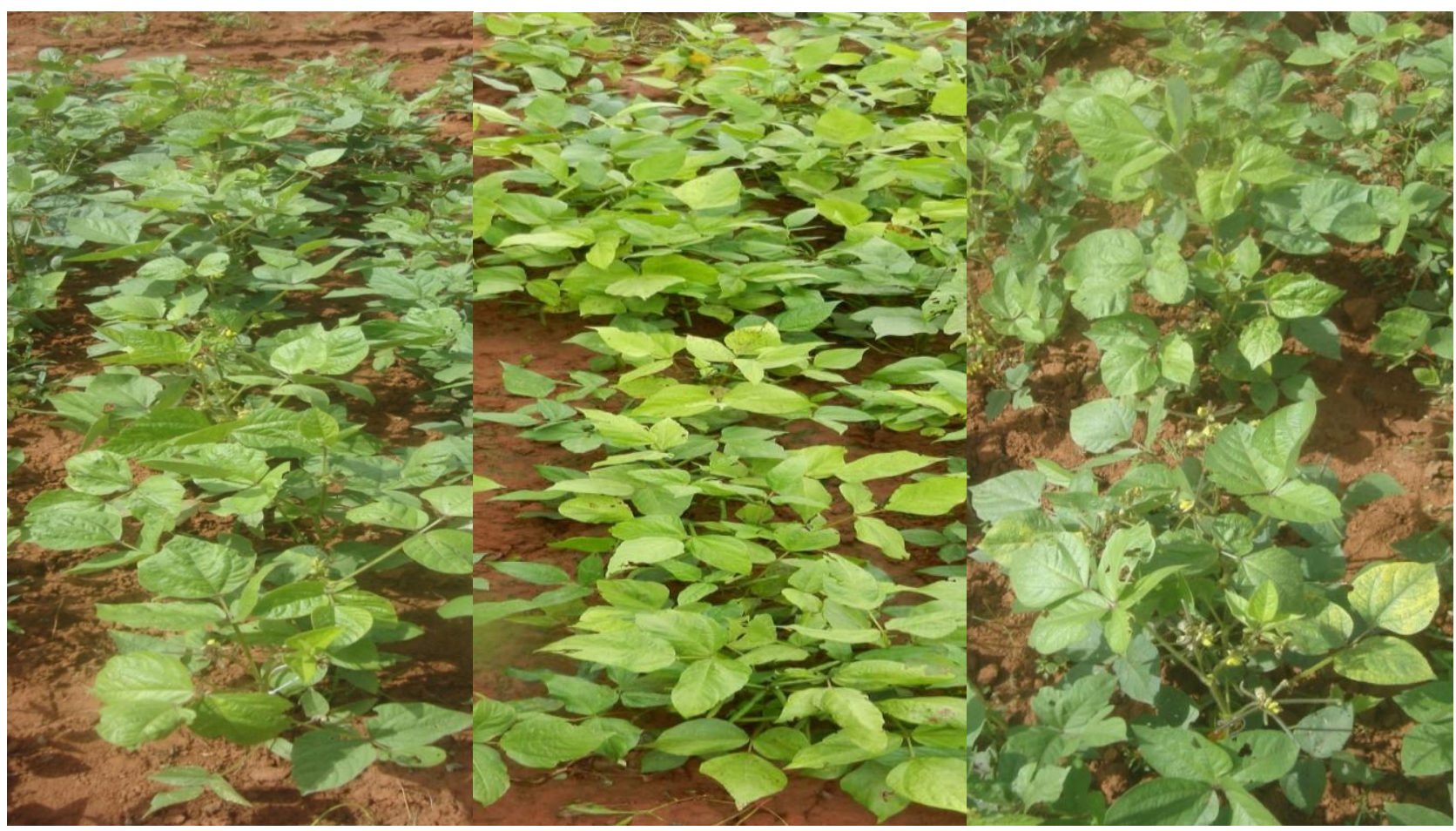

a) KMP-24

b) KMP-40

c) LGG-593 
In this study screened 126 mungbean germplasm lines against MYMV, among them 9 were highly susceptible. Present investigation showed that KKM-3 is susceptible to MYMV. However, Manjunath et al., (2013) reported KKM-3 as resistant cultivar against MYMV disease. It may be due to variability existing in vector and/or virus coupled with climate change. Among 64 mungbean lines evaluated under field conditions, 16 accessions were graded as susceptible whereas 7 were found highly susceptible (Munnawar et al., 2014). Mungbean genotypes such as KMP-13, KMP33, KMP-3, KMP-47, KMP-20, KMP-36, LGG-563, VGG-04-025, VGG-112, 2KM164, JNG-15and JNG-16 were found highly susceptible, showing disease reaction more than 50 per cent to MYMV. Sever yellow discolouration of leaves, stunting of plants, reduction in leaves and pod size were noticed. Salam (2011) and Singh et al., (2000) also found Chinamung was highly susceptible to MYMV.

\section{References}

Anonymous. 2012. Selected state wise Area, Production and Productivity of Moong (Kharif and Rabi) in India, Ministry of Agriculture and Farmers Welfare. Govt. of India.

Chatterjee, D. and Randhawa, G.S. 1952. Standardization of names of cultivated plants in India-11.Cereals, Pulses, Vegetables and spices. Indian J. Hort., 9: 64-84

Kang, B.C., Yeam, I. and Jahn, M.M. 2005. Genetics of plant virus resistance. Annu. Rev. Phytopathol., 43: 581621.
Manjunath, B., NeethaJayaram, Muniyappa, V. and Prameela, H. A., 2013.Status of yellow mosaic virus and whitefly Bemisia tabaci biotypes on mungbean in Southern Karnataka. Legume Res., 36(1): 62-66.

Mayee, C.D. and Datar, V.V. 1986. Phytopathometry, Marathawada Agricultural University, Parbhani. Technical Bull., No. 1, pp. 145-146.

Mohan, S., Sheeba, A.,Murugan, E. and Ibrahim, S.M. 2014. Screening of Mungbean germplasm for resistance to Mungbean Yellow Mosaic Virus under Natural Condition. Indian J. Sci. Technol., 7(7): 891-896.

Munnawar, M.H., Ali, A. and Malik, S.R. 2014. Identification of resistance in mungbean and mash bean germplasm against mungbean yellow mosaic virus. Pak. J. Agric., 27(2): 129-135.

Saikia, A.K. and Muniyappa, V. 1989. Epidemiology and control of tomato leaf curl virus in Southern India, Trop. Agric. (Trinidad)., 66: 350-354.

Salam, S.A. 2011. Studies on mungbean yellow mosaic virus disease on green gram. Karnataka J. Agric. Sci., 24(2): 247-248.

Singh, B.B. 2011. Project coordinators report. All India Coordinated Research Project on MULLaRP. Annual Group Meet; 1113 May 2011; Kanpur: Indian Council of Agricultural Research, Indian Institute of Pulses Research.

Singh, B.R., Chandra, S.S., Chandra, S. and Ram. S. 2000. Evaluation of mungbean varieties against yellow mosaic virus. Ann. Plant Prot. Sci., 8: 270-271.

Singh, K., Singh, S. and Gumber, R.K. 1996. Resistance to mungbean yellow mosaic virus in mungbean. Indian J. Pulses Res., 9(1): 90 .

\section{How to cite this article:}

Jayappa, H.K. Ramappa, Jabbar Sab, Chirag Gautam and B.D. Devamani. 2017. Screening of Mungbean Genotypes against Mungbean Yellow Mosaic Virus Disease. Int.J.Curr.Microbiol.App.Sci. 6(5): 1746-1752. doi: https://doi.org/10.20546/ijcmas.2017.605.189 